\title{
Research on the Evolution Path of Public Opinion in Environmental Emergencies
}

\author{
Kaixi Tan ${ }^{1, *}$, Lixun $X_{i e}^{1}$ and Lifa $\operatorname{Lin}^{1}$ \\ ${ }^{1}$ School of Geography and Bioinformatics, Nanjing University of Posts and Telecommunications, Nanjing,210023, China
}

\begin{abstract}
In recent years, environmental emergencies have spread rapidly on the social media, causing public concern. This study takes the "3.21" accident in Xiangshui, Jiangsu as an example, and obtains 46,265 Weibo comments by using Python program. After data preprocessing, 7740 Weibo comments were obtained. The evolution stage of public opinion about accidents is divided into outbreak period, temporary decline period, recurrence period and recession period by using the changing characteristics of public attention. This study uses the sentiment analysis model and LDA theme extraction model to analyze the emotional analysis and theme extraction of comments in each stage, analyze the emotional tendencies and Theme Evolution in each stage, and analyze the evolution law of public opinion in the "3.21" accident in Xiangshui, Jiangsu. Studies show that public attention decreases rapidly from the outbreak stage to the temporary decline stage, increases slightly in the recurrence stage, and lowest in the recession stage. Public sentiment is susceptible to derivative events throughout the evolution of public opinion. If the derivative events are encouraging events such as rescue and rescue, the proportion of public positive emotions will increase. If the derivative events are negative events such as corruption, the proportion of public negative emotions will increase. The research conclusion provides theoretical support for establishing public opinion response mechanism to environmental emergencies.
\end{abstract}

\section{Introduction}

With the popularity of the Internet in China, the social media platform based on Weibo has become the main channel for the public to access information. Because of the real-time and social nature of Weibo, the network public opinion triggered by environmental emergencies has been spread rapidly in the microblog platform, which makes the public keep a high degree of attention to the incident. If the public opinion is not guided in time, a large number of false reports and rumors will be generated. By studying the evolution path of public opinion in environmental contingencies, it is able to identify the mechanisms of occurrence, development, transmission and evolution of public opinion, thus mastering the dominance of public opinion.

At present, many scholars have done research on the evolution path of public opinion. The main research directions are divided into the stage of public opinion, emotional analysis and public opinion theory model research.

In the research of public opinion stage, Lin Ping, Liu Guowei, Gu Yiran, Dai Jianhua and so on use the LED model $^{[1]}$, Moran's I index and optimal segmentation theory ${ }^{[2]}$ and the modified $\mathrm{HK}$ model ${ }^{[3]}$ to study and analyze the evolution process of various types of public opinion events. In the area of emotional analysis, Pranamesh Chakraborty et al. used CoreNLP software to conduct emotional analysis, and built a public model of emotional changes in government decisions ${ }^{[4]}$. In the research of public opinion theory model, Jaewon Kim et al. constructed a network analysis model to quantitatively analyze the evolution of public opinion by comparing and analyzing the network data extracted from Twitter ${ }^{[5]}$.

In summary, most scholars use a single indicator to classify public opinion stages, and because of the small number of sample data and the complex sources, the public opinion evolution model is not accurate enough. In addition, there are relatively few studies on public opinion on environmental emergencies.

This paper selects several indicators, such as the number of tweets, comments and topics, to analyze the heat of public opinion events, to divide the evolution path of public opinion development stages, and to conduct emotional analysis, to study the emotional characteristics and theme changes in each stage. The results of the study are of great significance for the government to grasp the law of public opinion evolution and take targeted measures to control public opinion.

\section{Materials and Methods}

\subsection{Sentiment Analysis Model}

Considering the particularity and complexity of Weibo comments, this paper uses a corpus-based statistical 
machine learning method to judge the emotional orientation of the text to be tested. The best classifier for this case is MultionmialNB (Naive Bayesian classifier with polynomial distribution). The core formula of the Bayesian classifier is:

$$
\begin{array}{r}
P(Y \mid X)=\frac{P(X \mid Y) P(Y)}{P(X)} \\
P(X \mid Y)=P\left(X_{1}, X_{2}, \ldots, X_{\mathrm{n}} \mid Y\right) \\
=P\left(X_{1} \mid Y\right) P\left(X_{2} \mid Y\right) \ldots P\left(X_{\mathrm{n}} \mid Y\right)
\end{array}
$$

MultionmialNB assumes that the feature is generated from a simple polynomial distribution. Polynomial distribution can describe the frequency of occurrences of various types of samples, so polynomial Naive Bayesian. is well suited for describing the characteristics of occurrences or proportions of occurrences.

$\mathrm{P}(\mathrm{Y} \mid \mathrm{X})$ is called a posterior probability, $\mathrm{P}(\mathrm{Y})$ is called a prior probability, $\mathrm{P}(\mathrm{X} \mid \mathrm{Y})$ is called a likelihood probability, and $\mathrm{P}(\mathrm{X})$ is called evidence.

MultionmialNB assumes that the feature is generated from a simple polynomial distribution. Polynomial distribution can describe the frequency of occurrences of various types of samples, so polynomial Naive Bayesian is well suited for describing the characteristics of occurrences or proportions of occurrences.

\section{Results and Discussion}

Using "Jiangsu Xiangshui explosion" as the keyword to search the relevant microblogs after the incident of March 21, 2019, and got 46265 Weibo data. Each data includes user name, web address, microblog content, publishing time, likes, comments, forwarding number and other data attributes.

\subsection{Public Opinion Evolution Stage Division}

\subsubsection{Analysis on the Evolution of Public Concern}

Based on statistical analysis of Weibo data, the time distribution characteristics of microblog publications in the "3.21 Accident in Xiangshui, Jiangsu" for 35 days from March 21 to April 24, 2019 were obtained (Fig. 1).

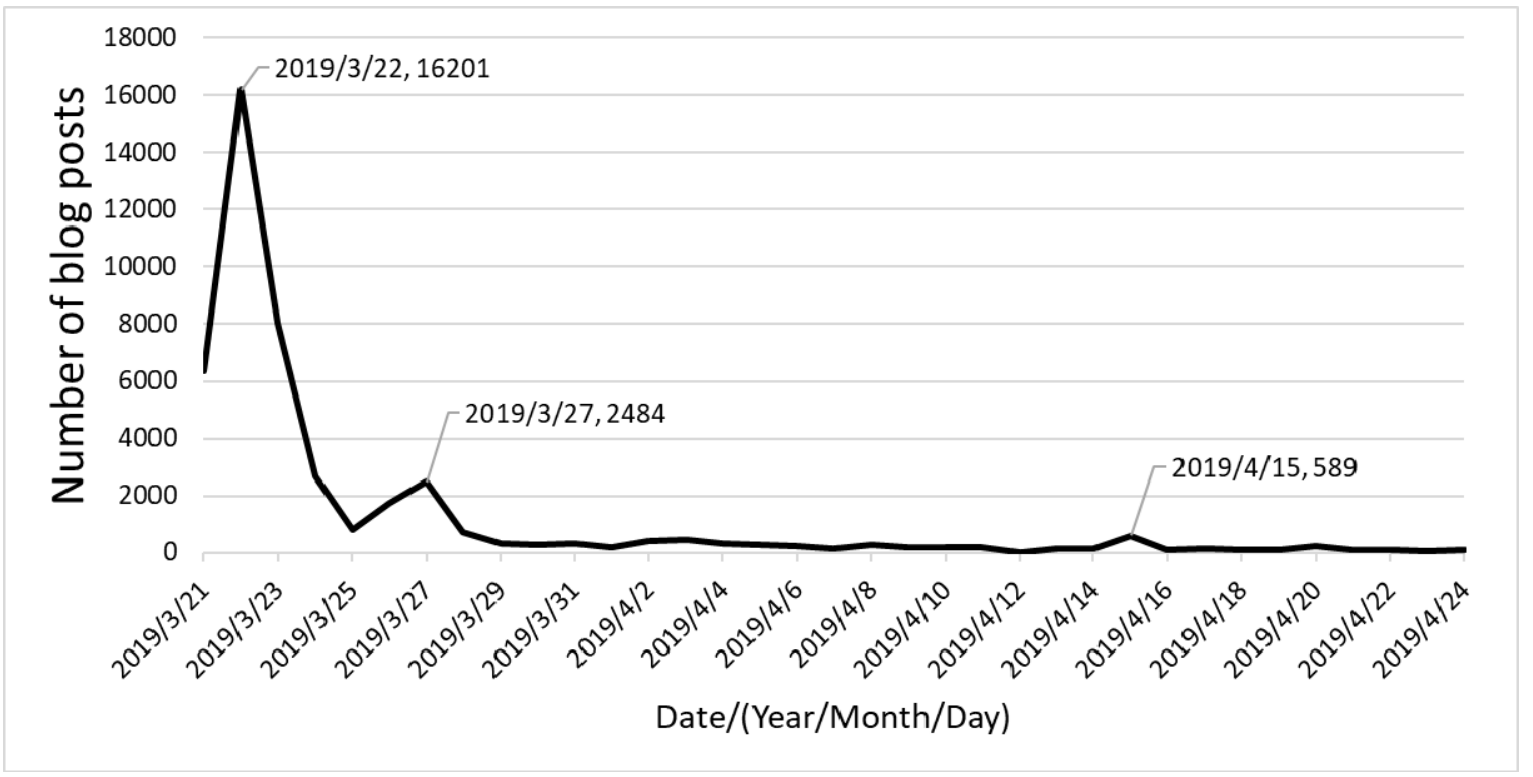

Figure 1. The change process of microblog publishing volume with time

The "Jiangsu Xiangshui 3.21" accident belongs to the type I of non-natural emergencies. The evolution of public opinion has the characteristics of long life cycle, multiple changes and multiple public opinion climaxes. Public blogging volume rises rapidly at the beginning of the incident and begins to decline after reaching its peak. There is periodic fluctuation during the decline, which conforms to the evolving characteristics of public opinion of type I non-natural emergencies (Figure 1).

The total number of microblogs posted represents the level of public interest in the event. At the beginning of the accident, that is, from March 21 to March 23, the public paid the highest attention to the incident, with an average daily blog volume of 10202, accounting for $66.16 \%$ of the total microblog in the study period. Since the main purpose of public microblogging is to spread the news about the accident, the main content of microblogging is focused on some basic information such as the time and location of the accident, as well as some queries, guesses and prayers.

Between March 24 and March 25, public attention to the incident declined rapidly due to the absence of official updates, with public blogs dropping to 827 on March 25.

Due to the influence of secondary public opinion of the accident, there were two minor outbreaks of public opinion on Weibo between March 26 to March 28 and April 15 to April 16. The content of the two stages of Weibo is also related to secondary public opinion.

After April 17, the public's attention to the accident gradually diminished because the related treatment of the 
accident was completed and there was no relevant secondary public opinion.

\subsubsection{Features and Causes of Public Opinion Turning Points}

The evolution graph of microblog spread shows that the evolution of public opinion in the "3.21" accident in Xiangshui, Jiangsu Province is complex, with three inflection points.

The first inflection point occurred on March 22, after a short burst of public opinion, which began to fade on March 22. After the accident, due to the convenience, speed and rapidity of the Internet, public opinion erupted rapidly in a short time. After a brief public outbreak, public interest in the incident quickly began to wane because no new information was generated about it.

The second inflection point occurred on March 25, and after a short recession, public opinion erupted again after March 25. The reason is that the search and rescue work ended on March 25 and the casualties of the accident (164 people were successfully rescued, 78 people died and 86 people survived) were announced. Some events in the search and rescue process caused public debate on the Internet.

The third inflection point occurred on April 15, which was caused by the compulsory measures taken by relevant agencies on April 15 to suspect the accident, which caused a small outbreak of public opinion again. Netizens expressed their opinions one after another. On that day, the amount of public blogs rose to 589, more than four times that of the previous day.

\subsubsection{Analysis of Public Opinion Evolution Stage}

According to the evolution model of public opinion path of environmental emergencies proposed in this paper, combined with the evolution of network public opinion of the "3.21" accident in Xiangshui, Jiangsu Province, it is divided into four stages: outbreak stage, temporary decline stage, recurrence stage and recession stage. The specific stages are divided into: outbreak stage: March 21-March 23; temporary decline stage: March 24-March 25; recurrence stage: March 26-April 15; regression stage: April 16-April 24.

\subsection{Analysis of Emotional Evolution}

Emotional propensity is an important feature of public opinion. It is greatly significant to conduct an analysis of emotional propensity in the study of public opinion, which helps to study the public opinion attitudes of netizens. Using the MultionmialNB model, this paper discriminates the affective tendency of 7740 preprocessed data of microblog texts about the "3.21" accident in Xiangshui, Jiangsu Province" from March 21 to May 15, 2009, and divides them into positive and negative emotions (Fig. 2).

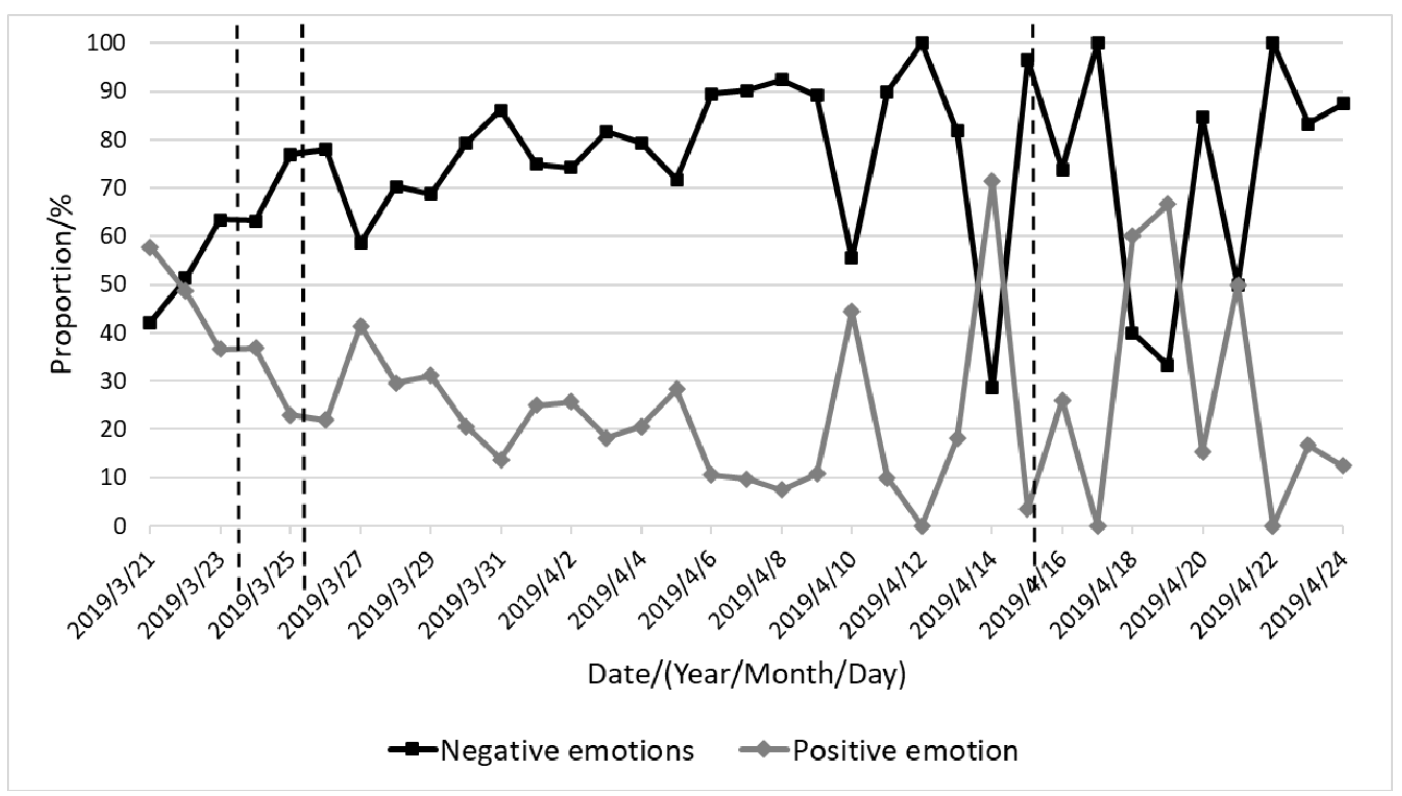

Figure 2. Time course of the proportion of each type of emotion

As can be seen from Figure 2, in the process of public opinion development, the public's emotional tendencies change greatly with time, indicating the characteristics of public opinion development, that is, the public's emotional tendencies are susceptible to many factors.

In the early stage of public opinion development (i.e. the period of public outbreak), public attention is high, positive and negative emotions are strong, and within three days of the incident, public emotional tendencies change significantly. The specific manifestations are as follows: on the day of the accident, due to the sudden occurrence and lack of information, the public's focus is on the accident itself, most of the content published is prayer and guess about the accident, so the proportion of the public with positive emotions is larger, accounting for $57.7 \%$ of the total attention, the public mood is more moderated, and the overall mood of society is more 
stable. On the second and third days after the accident happened, as more negative information about the accident (such as workers' casualties, kindergartens near chemical factories, etc.) was distributed, the average public sentiment gradually developed to negative. On March 23, the proportion of positive emotions was only $36.6 \%$, and the overall social sentiment began to develop in a negative direction.

During the temporary decline period of public opinion, that is, from March 24 to March 25, the positive and negative emotional intensity of the public decreased slightly compared with the outbreak period. The proportion of positive emotions among the public was still low, only $39.8 \%$. During this period, the public had a more distinct position. They expressed their views on the accident, mostly negative emotions, and the public was more excited. In this stage, some netizens will have the mentality to follow the trend and easily lose their senses, while pushing the network public opinion to a more severe direction, so the government's positive guidance of public opinion is especially needed in this stage.

During the period of public opinion recurrence, that is, from March 26 to April 15, the public's positive and negative emotional intensity caused by the secondary public opinion of the accident fluctuated to some extent, but the overall emotional emphasis was at a low level. Most of the time, the average value of social emotions was negative. The proportion of positive public emotions was further reduced from the previous stage, accounting. for only $25.5 \%$ of the total emotions. The reason is that during this period, news about accident casualties and the disposition of criminal suspects was gradually published, which caused a lot of public dissatisfaction. At this time, the public mood is fierce, which easily induces netizens to sympathize with the victims and hate public rights. This situation easily leads to the uncontrolled development of public opinion, which needs the correct guidance of the government and mainstream media.

During the period of public opinion recession, from April 16 to April 24, the emotional intensity decreased to a very low level. Although social emotions still tend to be negative, due to the basic completion of the accident handling, netizens' attention to the accident declined and the general public mood stabilized.

\section{Conclusions}

This study takes the "3.21" accident in Xiangshui, Jiangsu as an example, to analyze the evolution path of public opinion and public sentiment of environmental accidents, and the results show that:

(1) Public attention of environmental emergencies decreases cliff-like from the outbreak stage to the temporary decline stage, increases slightly from the repetitive stage to the recession stage, and is extremely low. On the basis of the law, it is very important for the government to intervene at the first time, grasp the dominant power of public opinion, actively respond to public demands, shape public confidence and fill the information vacuum.

(2) Public sentiment is susceptible to derivative events throughout the evolution of public opinion. If the derivative events are inspiring events such as rescue and rescue, the proportion of public positive emotions will increase. If the derivative events are negative events such as inadequate supervision, the proportion of public negative emotions will increase. This has a major bearing on the government's work in disaster prevention and control in peacetime.

\section{Acknowledgments}

This paper is one of the phased results of the National Undergraduate Innovation and entrepreneurship training project (project number: 202010293032Z).

\section{References}

1. Lin P., Huang W. (2010) Event Topic Evolution of Network Public Opinions: An Analysis Based on LDA Model. J. Journal of Intelligence, 30: 26-30.

2. Liu G., Cheng G., Jiang J. (2015) On the Evolution of the Unconventional Emergency Network Public Opinion from the Perspective of Spatial-temporal Differentiation— Taking "Shanghai 12. 31 Stampede" as an Example. J. Journal of Intelligence, 34: $126-130+150$

3. Jianhua Dai, Yanan Li. Modeling and Simulating of Network Public Opinion Evolution Based on Dynamic Reference Point of Prospect Theory. 2017.

4. Chakraborty P, Sharma A. Public Opinion Analysis of the Transportation Policy Using Social Media Data: A Case Study on the Delhi Odd-Even Policy $[\mathrm{J}]$. Transportation in Developing Economies, 2019, 5(1).

5. Kim J. Analyzing public opinion toward the 2019 North Korea-United States summit through mining twitter $[\mathrm{J}]$. Proceedings of the Association for Information Science and Technology, 2019, 56(1): 686-687. 